\title{
THE ACIDOTIC STATE OF NORMAL NEW-BORNS
}

\author{
WITH SPECIAL REFERENCE TO THE ALVEOLAR $\mathrm{CO}_{2}$ TENSION, ALKALI \\ TOLERANCE AND ACETONURIA \\ MAX SEHAM, M.D. \\ Instructor in Pediatrics, University of Minnesota, Medical School \\ MINNEAPOLIS
}

The work of Ylippo, in 1914, was a stimulus to the study of this subject in new-borns. He claims to have found (1) that the human organism at birth is in a state of "physiologic acidosis"; (2) that the blood shows an "acidotic constitution" (he having found the $\mathrm{P}_{\mathrm{H}}$ of the blood to be as low as 6.58); (3) that the $\mathrm{CO}_{2}$ dissociation curve of the blood of new-borns shows an acidosis (20 per cent. less than in adults), and (4) that the hydrogen concentration is uniformly higher than in adults. Of the many methods for determining acidosis, the two most accurate are, first, the chemical study of the blood, and second, the study of the $\mathrm{CO}_{2}$ alveolar tension. The method for determining the $\mathrm{CO}_{2}$ alveolar tension is simple and in most conditions an accurate one for determining acidosis. So far as we know this has never been determined in new-borns.

Alveolar air is the air which is in or comes from the alveolae of the lungs. As the exchange of $\mathrm{CO}_{2}$ between the lungs and the blood takes place in the alveolae, the tension of the different gases in the alveolae (according to the law of difftusion of gases) will be practically the same as in the blood. The percentage of $\mathrm{CO}_{2}$ in the alveolar air is not quite the same in different individuals, but the average is about 5.6 for adults. This in terms of pressure is about $40 \mathrm{~mm}$.

Technic.-One hundred and fifty-one determinations were made on fifty babies. The youngest was one hour old, the oldest was 32 weeks of age. The majority were from one day to one week old, and there were two premature babies, one of seven and one eight months. The effects of food and exercise (crying and struggling) on the $\mathrm{CO}_{2}$ tension were studied. Of the various methods of obtaining alveolar air, it was necessary to choose the method most easily adapted to the new-born.

Haldane's method is, of course, impractical because with this the cooperation of the patient is necessary; the same objection applies to Lindhard's method. Higgins' modification of Plesch's method is by far the most advantageous in working with new-borns. Instead of using the Hygeia nipple with rubber tubing over the mouth, as is suggested by Howland ${ }^{1}$ for infants, the pulmotor mask for new-borns

1. Howland, J. H., and Marriott, W. McK.: Acidosis Occurring with Diarthea, Am. J. Dis. Child. 11:309, 1916. 
which could be applied very snugly to the face, preventing any escape of air, was used. The principle of this method of collecting air is quite different from the Haldane. Instead of trying to obtain samples of air from the alveolae, by means of deep respirations, this method necessitates the rebreathing of a certain amount of air until it is in equilibrium with the air in the alveolae. The initial amount of air in the bag, the length of time for breathing, and the character of the breathing, must be considered, in order to determine the results accurately. The greatest difficulty met with in securing uniform readings was that the character and rate of breathing could not be controlled.

The pulmotor mask has two brass tubes; the upper one is used to allow the baby to breathe through before beginning the experiment, after the mask has been put in place; the lower tube has a three-way valve and is connected to the football bag by another rubber tube. It is preferable to begin the experiment at the end of an expiration and to end it at the beginning of an inspiration, but this, of course, with new-borns is usually impossible. It was necessary to examine the air in the bag immediately because diffusion takes place very readily, and the readings are lower in the fourth and fifth specimens than in the first ones.

TABle 1.-Determinations of $\mathrm{CO}_{2}$ Alveolar Tension of NORMAL NEW-BORNS

(Time of Reading, 30 Seconds. Initial Air in Bag 150 to 250 c.c.; Average, 200 c.c.)

\begin{tabular}{|c|c|c|c|c|c|c|c|c|}
\hline No. & Date & $\underset{\text { Day }}{\text { Time of }}$ & Age & $\begin{array}{l}\text { Air in } \\
\text { Bag. } \\
\text { C.c. }\end{array}$ & $\begin{array}{l}\text { Time, } \\
\text { Sec. }\end{array}$ & $\begin{array}{c}\text { Hal- } \\
\text { dane } \\
\text { Method }\end{array}$ & $\begin{array}{l}\text { Mar- } \\
\text { riott } \\
\text { Method }\end{array}$ & Remarks \\
\hline 1 & $12 / 13$ & $9: 30$ a.m. & 3 days & 150 & 30 & 24.6 & 22.0 & $\begin{array}{l}\text { Cried twice before } \\
\text { reading }\end{array}$ \\
\hline 2 & $12 / 14$ & 1:00 p.m. & 2 days & 150 & 30 & 22.38 & 18.0 & Quiet \\
\hline 3 & $12 / 14$ & 1:10 p.m. & 11 days & 200 & 30 & $\cdots \cdots$ & $\begin{array}{l}18.0 \\
22.0\end{array}$ & $\begin{array}{l}\text { Cried and struggled } \\
\text { during reading }\end{array}$ \\
\hline 4 & $12 / 14$ & 1:20 p.m. & 5 days & 200 & 30 & 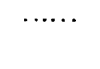 & $\begin{array}{l}18.0 \\
22.0\end{array}$ & $\begin{array}{l}\text { Cried ten times before } \\
\text { but quiet during } \\
\text { reading }\end{array}$ \\
\hline 5 & $12 / 14$ & 1:45 p.m. & 5 days & 150 & 30 & $\cdots \cdots$ & $\begin{array}{l}19.0 \\
20.0\end{array}$ & $\begin{array}{l}\text { Cried ten times but } \\
\text { quiet during reading }\end{array}$ \\
\hline f & $12 / 14$ & 2:15 p.m. & 3 days & 150 & 30 & $\cdots \cdots$ & $\begin{array}{l}22.0 \\
20.0\end{array}$ & $\begin{array}{l}\text { Cried flve times be- } \\
\text { fore reading }\end{array}$ \\
\hline 7 & $12 / 14$ & 4:00 p.m. & 3 days & 150 & 30 & $\ldots \ldots$ & $\begin{array}{l}18.0 \\
20.0\end{array}$ & Cried during reading \\
\hline 8 & $12 / 17$ & 4:00 p.m. & 6 days & 150 & 30 & . & $\begin{array}{l}23.0 \\
25.0\end{array}$ & Quiet \\
\hline 9 & $12 / 20$ & 1:40 p.m. & 7 days & 150 & 30 & $\begin{array}{l}32.08 \\
34.32\end{array}$ & $\begin{array}{l}25.0 \\
27.0\end{array}$ & Quiet \\
\hline 10 & $12 / 20$ & 1:50 p.m. & 10 days & 150 & 30 & $\begin{array}{l}25.36 \\
24.62\end{array}$ & $\begin{array}{l}25.0 \\
23.0\end{array}$ & Quiet \\
\hline 11 & $12 / 20$ & 1:55 p.m. & 4 days & 150 & 30 & $\begin{array}{l}28.35 \\
28.94\end{array}$ & $\begin{array}{l}220 \\
25.0\end{array}$ & Quiet \\
\hline 12 & $12 / 21$ & 10:30 a.m. & 8 days & 150 & 30 & $\begin{array}{l}35.06 \\
35.8\end{array}$ & $\begin{array}{l}33.0 \\
35.0\end{array}$ & \\
\hline 13 & $12 / 21$ & 11:45 a.m. & 5 days & 150 & 30 & $\begin{array}{l}35.8 \\
27.6\end{array}$ & $\begin{array}{l}20.0 \\
33.0\end{array}$ & \\
\hline 14 & $12 / 21$ & 1:30 p.m. & 8 days & 150 & 30 & $\begin{array}{l}24.6 \\
26.11\end{array}$ & $\begin{array}{l}26.0 \\
30.0\end{array}$ & \\
\hline 15 & $12 / 21$ & 1:45 p.m. & 5 hrs. & 150 & 30 & 22.30 & 20.0 & . \\
\hline
\end{tabular}


TABLE 1.-Determinations of $\mathrm{CO}_{2}$ Alveolar Tension of Normal New-Borns-(Continued)

(Time of Reading, 30 Seconds. Initial Air in Bag 150 to 250 c.c.; Average, 200 c.c.)

\begin{tabular}{|c|c|c|c|c|c|c|c|c|}
\hline No. & Date & $\underset{\text { Day }}{\text { Time of }}$ & Age & $\begin{array}{l}\text { Air in } \\
\text { Bag, } \\
\text { C.c. }\end{array}$ & $\begin{array}{l}\text { Time, } \\
\text { Sec. }\end{array}$ & $\begin{array}{c}\text { Hal- } \\
\text { dane } \\
\text { Method }\end{array}$ & $\begin{array}{c}\text { Mar- } \\
\text { iott } \\
\text { Method }\end{array}$ & Remarks \\
\hline 16 & $12 / 21$ & 2:00 p.m. & 3 hrs. & 150 & 30 & $\begin{array}{l}23.87 \\
27.6\end{array}$ & $\begin{array}{l}20.0 \\
29.0\end{array}$ & \multirow{13}{*}{$\begin{array}{l}\text { Very quiet } \\
\text { Orying, struggling } \\
\text { Bleeder }\end{array}$} \\
\hline 17 & $1 / 29$ & 3:40 p.m. & 4 days & 150 & 30 & 32.08 & 22.0 & \\
\hline 18 & $1 / 29$ & 4:00 p.m. & 7 days & 150 & 30 & $\begin{array}{l}35.8 \\
29.84\end{array}$ & $\begin{array}{l}25.0 \\
27.0\end{array}$ & \\
\hline 19 & $2 / 2$ & 10:30 a.m. & 2 mos. & 150 & 30 & 26.8 & $\begin{array}{l}30.0 \\
35.0\end{array}$ & \\
\hline 20 & $2 / 2$ & 11:15 a.m. & 4 hrs. & 150 & 30 & $\begin{array}{l}35.8 \\
25.8\end{array}$ & $\begin{array}{l}40.0 \\
28.0\end{array}$ & \\
\hline 21 & 2/ 7 & 9:45 a.m. & $2 \mathrm{mos}$ & 150 & & $\begin{array}{l}26.11 \\
28.35\end{array}$ & $\begin{array}{l}30.0 \\
35.0\end{array}$ & \\
\hline & & & & & & 21.63 & 32.0 & \\
\hline 22 & $2 / 7$ & 10:05 a.m. & 1 day & 150 & 30 & $\begin{array}{l}27.6 \\
29.8\end{array}$ & $\begin{array}{l}28.0 \\
30.0\end{array}$ & \\
\hline 23 & $2 / 9$ & 12:10 p.m. & 2 mos. & $1 E 0$ & 30 & $\begin{array}{l}37.13 \\
34.3\end{array}$ & $\begin{array}{l}25.0 \\
30.0\end{array}$ & \\
\hline 24 & $2 / 10$ & 1:00 p.m. & 1 day & 150 & 30 & $\begin{array}{l}32.8 \\
31.3\end{array}$ & $\begin{array}{l}25.0 \\
22.0\end{array}$ & \\
\hline 25 & $2 / 13$ & 2:50 p.m. & 2 days & 150 & 30 & $\begin{array}{l}\mathbf{2 2 . 3} \\
\mathbf{2 0 . 8}\end{array}$ & $\begin{array}{l}20.0 \\
25.0\end{array}$ & \\
\hline 26 & $2 / 13$ & 3:45 p.m. & 7 days & 150 & 30 & $\begin{array}{l}29.8 \\
34.3\end{array}$ & $\begin{array}{l}20.0 \\
25.0\end{array}$ & \\
\hline 27 & $2 / 13$ & $10: 40$ a.m. & 1 day & 150 & 30 & $\begin{array}{l}22.38 \\
25.36\end{array}$ & & \\
\hline 28 & $2 / 14$ & $10: 50$ a.m. & 5 days & 150 & 30 & $\begin{array}{l}41.7 \\
42.5\end{array}$ & $\begin{array}{l}25.0 \\
30.0\end{array}$ & \\
\hline 29 & $2 / 14$ & 11:35 a.m. & 8 days & 150 & 30 & $\begin{array}{l}39.5 \\
37.3\end{array}$ & $\begin{array}{l}25.0 \\
28.0\end{array}$ & \\
\hline 30 & $2 / 14$ & Noon & 1 day & 150 & 30 & $\begin{array}{l}27.0 \\
27.0 \\
22.0\end{array}$ & $\begin{array}{l}20.0 \\
20.0\end{array}$ & \\
\hline 31 & $2 / 15$ & 11:40 a.m. & 8 days & 150 & 30 & $\begin{array}{l}35.06 \\
37.3\end{array}$ & $\begin{array}{l}25.0 \\
28.0\end{array}$ & \\
\hline 32 & $2 / 15$ & $11: 40$ a.m. & 1 day & 150 & 30 & $\begin{array}{l}22.3 \\
22.3\end{array}$ & $\begin{array}{l}30.0 \\
28.0\end{array}$ & \\
\hline 33 & $2 / 15$ & Noon & $18 \mathrm{hrs.}$ & 150 & 30 & $\begin{array}{l}27.6 \\
28.3\end{array}$ & & \\
\hline 34 & $2 / 17$ & Noon & 3 days & 150 & 30 & $\begin{array}{l}31.3 \\
29.8\end{array}$ & $\begin{array}{l}25.0 \\
27.0\end{array}$ & \\
\hline 35 & $2 / 17$ & 1:00 p.m. & 3 days & 150 & 30 & $\begin{array}{l}27.6 \\
26.8\end{array}$ & 25.0 & \\
\hline 36 & $2 / 17$ & 1:30 p.m. & 8 days & 150 & 30 & $\begin{array}{l}35.06 \\
35.06\end{array}$ & 35.0 & - \\
\hline 37 & $2 / 17$ & 1:30 p.m. & 1 day & 150 & 30 & $\begin{array}{l}24.6 \\
22.8\end{array}$ & 20.0 & \\
\hline 38 & $2 / 18$ & 11:10 a.m. & 2 days & 150 & 30 & $\begin{array}{l}39.5 \\
42.5\end{array}$ & 20.0 & Collanse \\
\hline 39 & $2 / 18$ & 11:30 a.m. & 4 days & 150 & 30 & $\begin{array}{l}29.8 \\
29.8\end{array}$ & 25.0 & Comapse \\
\hline 40 & $2 / 18$ & $\begin{array}{r}\text { 12:15 p.m. } \\
\text { 1:10 p.m. }\end{array}$ & $\begin{array}{c}2 \text { days } \\
\ldots \ldots \ldots\end{array}$ & $\begin{array}{l}150 \\
\ldots\end{array}$ & $\begin{array}{l}30 \\
\ldots\end{array}$ & $\begin{array}{l}23.1 \\
23.8\end{array}$ & & \\
\hline 41 & $2 / 19$ & 12:45 p.m. & 4 days & 150 & 30 & $\begin{array}{l}23.8 \\
22.3\end{array}$ & $\begin{array}{l}30.0 \\
32.0\end{array}$ & \\
\hline 42 & $2 / 19$ & 1:20 p.m. & 4 days & 150 & 30 & $\begin{array}{l}20.14 \\
22.3\end{array}$ & $\begin{array}{l}25.0 \\
30.0\end{array}$ & \\
\hline 43 & $2 / 20$ & 1:20 p.m. & 9 days & 150 & 30 & $\begin{array}{l}23.13 \\
23.13\end{array}$ & $\begin{array}{l}25.0 \\
\ldots \ldots\end{array}$ & Collapse \\
\hline 44 & $2 / 23$ & 1:45 p.m. & 1 day & 150 & 30 & $\begin{array}{l}24.6 \\
24.6\end{array}$ & 20.0 & \\
\hline 45 & $2 / 25$ & 3:00 p.m. & 2 hrs. & 150 & 30 & $\begin{array}{l}28.3 \\
18.0-\end{array}$ & $\cdots \cdots$ & Too quiet breathing \\
\hline 46 & $2: 25$ & 3:30 p.m. & 3 days & 150 & 30 & $\begin{array}{l}37.3 \\
37.3\end{array}$ & 30.0 & \\
\hline 47 & $2 / 25$ & 4:00 p.m. & $1 \mathrm{hr}$. & 150 & $\mathbf{3 0}$ & $\begin{array}{l}26.11 \\
25.36\end{array}$ & 20.0 & \\
\hline 48 & $2 / 25$ & 4:30 p.m. & 6 days & 150 & 30 & $\begin{array}{l}35.06 \\
36.5\end{array}$ & & \\
\hline 49 & $2 / 25$ & 5:00 p.m. & 1 day & 150 & 30 & $\begin{array}{l}12.6 \\
20.0\end{array}$ & 15.0 & Very quiet \\
\hline 50 & $2 / 25$ & 5.15 p.m. & 1 day & 150 & 30 & $\begin{array}{l}16.41 \\
18.65\end{array}$ & 20.0 & \\
\hline
\end{tabular}


In Table 1, fifty readings were made on twenty normal new-borns. The time of reading was the standard 30 seconds, which is the time of circulatory cycle. The initial amount of air in the bag was 150 to 250 .c.c., the average being 200 c.c. This was taken according to Howland's technic with infants.

The babies were from one hour to two months old. It can be seen that the readings with Marriott's method are lower than with Haldane's. This may be because Marriott's was done after Haldane's, the $\mathrm{CO}_{2}$ tension becoming lower on account of escape of air, from bag.

The results by Haldane's ranged from about 20 to $42 \mathrm{~mm}$. The average reading was $30 \mathrm{~mm}$. Readings were made at about the same time each day on the same babies and under the same conditions. From these fifty readings no conclusions could be drawn, the results varying too much.

TABle 2.-Determinations of $\mathrm{CO}_{2}$ Alveolar Tension in Twenty

NORMAL NEW-BORNS

(Time of Reading, 30 Seconds. Air in Bag, 50 c.c.)

\begin{tabular}{|c|c|c|c|c|c|c|c|}
\hline No. & Date & $\underset{\text { Day }}{\text { Time of }}$ & Age & $\begin{array}{c}\text { Air in } \\
\text { Bag, } \\
\text { C.e. }\end{array}$ & $\begin{array}{c}\text { Time, } \\
\text { Breath, } \\
\text { Sec. }\end{array}$ & $\begin{array}{c}\text { Hal- } \\
\text { d:?ne } \\
\text { Method }\end{array}$ & Remarks \\
\hline 1 & $3 / 9$ & 7:00 p.m. & 5 days & 50 & 30 & $\begin{array}{l}35.0 \\
33.57\end{array}$ & \\
\hline 2 & $3 / 9$ & 5:20 p.m. & 9 days & 50 & 30 & $\begin{array}{l}40.28 \\
40.28\end{array}$ & \\
\hline 3 & $3 / 9$ & 5:20 p.m. & 5 days & 50 & 30 & $\begin{array}{l}34.32 \\
36.33\end{array}$ & \\
\hline 4 & $3 / 8$ & 5:15 p.m. & 4 days & 50 & 30 & $\begin{array}{l}24.6 \\
28.3\end{array}$ & After; very quiet \\
\hline 5 & $3 / 8$ & 5:00 p.m. & 8 days & $\tilde{80}$ & 30 & $\begin{array}{l}31.33-? \\
40.28\end{array}$ & After \\
\hline 6 & $3 / 8$ & $4: 10$ p.m. & 4 days & 50 & 30 & $\begin{array}{l}42.52 \\
41.78\end{array}$ & After \\
\hline 7 & $3 / 8$ & 1:00 p.m. & 5 days & 50 & 30 & $\begin{array}{l}37.3 \\
39.5\end{array}$ & Before cried \\
\hline 8 & $3 / 8$ & 12 Noon & 5 days & 50 & 30 & $\begin{array}{l}43.2 \\
42.5\end{array}$ & After struggled \\
\hline $\boldsymbol{9}$ & $3 / 7$ & 2:30 p.m. & 4 days & 50 & 30 & $\begin{array}{l}26.11 \\
28.35\end{array}$ & After cried \\
\hline 10 & $3 / 7$ & 4:50 p.m. & 7 days & 50 & 30 & $\begin{array}{l}44.01 \\
44.76\end{array}$ & After quiet \\
\hline 11 & $3 / 7$ & 4:40 p.m. & 6 hrs. & 50 & 30 & $\begin{array}{l}39.54 \\
40.28\end{array}$ & After cried \\
\hline 12 & $3 / 7$ & 4:30 p.m. & 3 days & 50 & 30 & $\begin{array}{l}37.3 \\
41.03\end{array}$ & After cried \\
\hline 13 & $3 / 7$ & 2:00 p.m. & 4 days & 50 & 30 & $\begin{array}{l}43.2 \\
44.01\end{array}$ & Before vomited, struggled \\
\hline 14 & $3 / 7$ & 1:50 p.m. & 7 days & 50 & 30 & $\begin{array}{l}41.03 \\
41.03\end{array}$ & \\
\hline 15 & $3 / 7$ & 1:10 p.m. & 7 days & 50 & 30 & $\begin{array}{l}31.3 \\
37.3\end{array}$ & Before cried \\
\hline 16 & $3 / 7$ & 12:30 p.m. & 3 days & 50 & 30 & $\begin{array}{l}36.5 \\
39.5\end{array}$ & After \\
\hline 17 & $3 / 7$ & $12: 10 \mathrm{p} . \mathrm{m}$ & 7 days & 50 & 30 & $\begin{array}{l}42.5 \\
40.28\end{array}$ & After \\
\hline 18 & $3 / 6$ & 2:10 p.m. & $\cdots \cdots \cdots$ & 50 & 30 & $\begin{array}{l}41.78 \\
43.27\end{array}$ & Before quiet \\
\hline 19 & $3 / 6$ & 1:10 p.m. & 3 days & 50 & 30 & $\begin{array}{l}35.0 \\
37.3\end{array}$ & Before quiet \\
\hline 20 & $3 / 5$ & 12 Noon & 5 days & 50 & 30 & $\begin{array}{l}38.79 \\
41.03\end{array}$ & After quiet \\
\hline 21 & $3 / 5$ & 11:15 a.m. & $1 \mathrm{day}$ & 50 & 30 & $\begin{array}{l}35.06 \\
37.3\end{array}$ & After cried \\
\hline
\end{tabular}


Table 2.-Determinations of $\mathrm{CO}_{2}$ Alveolar Tension in Twenty Normal New-Borns-(Continued)

(Time of Reading, 30 Seconds. Air in Bag, 50 c.c.)

\begin{tabular}{|c|c|c|c|c|c|c|c|}
\hline No. & Date & $\underset{\text { Day }}{\text { Time of }}$ & Age & $\begin{array}{l}\text { Air in } \\
\text { Bag, } \\
\text { C.c. }\end{array}$ & $\begin{array}{c}\text { Time, } \\
\text { Breath, } \\
\text { Sec. }\end{array}$ & $\begin{array}{c}\text { HaI- } \\
\text { dane } \\
\text { Method }\end{array}$ & Remarks \\
\hline 22 & $3 / 5$ & 10:00 a.m. & 3 days & 50 & 30 & 35.8 & Before quiet \\
\hline 23 & $3 / 3$ & 11:00 a.m. & $1 \mathrm{day}$ & 50 & 30 & 44.76 & Struggled after \\
\hline 24 & $3 / 4$ & 5:00 p.m. & 4 days & 50 & 30 & 46.25 & Before quiet \\
\hline 25 & $3 / 4$ & 4:45 p.m. & 1 day & 50 & 30 & 42.5 & Quiet before \\
\hline 26 & $3 / 4$ & 4:00 p.m. & 4 days & 50 & 30 & 45.5 & \\
\hline 27 & $3 / 4$ & 3:30 p.m. & $11 \mathrm{hrs}$. & 50 & 30 & $\begin{array}{l}28.35 \\
26.11\end{array}$ & Barely breathed \\
\hline 28 & $3 / 3$ & 5:15 p.m. & $1 \mathrm{day}$ & 50 & 30 & 41.78 & Before quiet \\
\hline 29 & $3 / 3$ & 5:15 p.m. & 3 days & 50 & 30 & $\begin{array}{l}40.28 \\
37.3\end{array}$ & After quiet \\
\hline 30 & $3 / 3$ & 4:50 p.m. & 3 days & 50 & 30 & $\begin{array}{l}32.08 \\
30.59\end{array}$ & After cried \\
\hline 31 & $3 / 3$ & 4:45 p.m. & 10 hrs. & 50 & 30 & $\begin{array}{l}45.51 \\
44.01\end{array}$ & After quiet \\
\hline 32 & $3 / 3$ & 9:50 a.m. & 4 hrs. & 50 & 30 & $\begin{array}{l}42.52 \\
43.27\end{array}$ & Before struggled very much \\
\hline 33 & $3 / 3$ & 10:20 a.m. & $\ldots \ldots$ & 60 & 30 & $\begin{array}{l}\text { 38.09 } \\
37.3\end{array}$ & Before cried very much \\
\hline 34 & $3 / 3$ & 10:00 a.m. & $\cdots$ & 50 & 30 & $\begin{array}{l}36.5 \\
35.8\end{array}$ & $\begin{array}{l}\text { Be fore quiet, shallow } \\
\text { breathing }\end{array}$ \\
\hline 35 & $3 / 3$ & 9:00 a.m. & ........ & 50 & 30 & $\begin{array}{l}45.5 \\
45.5\end{array}$ & Before quiet \\
\hline 36 & $3 / 3$ & 9:50 a.m. & ........ & 60 & $\mathbf{3 0}$ & $\begin{array}{l}45.51 \\
45.51\end{array}$ & Before quiet \\
\hline 37 & $3 / 3$ & 9:40 a.m. & 3 days & 50 & 30 & $\begin{array}{l}43.27 \\
42.52\end{array}$ & Before quiet \\
\hline 38 & $3 / 2$ & 3:00 p.m. & 5 hrs. & 50 & 30 & $\begin{array}{l}40.28 \\
36.55\end{array}$ & Before quiet \\
\hline 39 & $3 / 2$ & 3:00 p.m. & ....... & 50 & 50 & $\begin{array}{l}35.06 \\
29.89\end{array}$ & $\begin{array}{l}\text { Before quiet; can't explain } \\
\text { after }\end{array}$ \\
\hline 40 & $3 / 2$ & 10:00 a.m. & $\cdots$ & 50 & 30 & $\begin{array}{l}41.28 \\
44.01\end{array}$ & Before cried \\
\hline 41 & $3 / 2$ & 10:00 a.m. & 1 hour & 50 & 30 & $\begin{array}{l}38.7 \\
35.9\end{array}$ & \\
\hline $\begin{array}{l}42 \\
43\end{array}$ & $\begin{array}{l}3 / 2 \\
3 / 1\end{array}$ & $\begin{array}{r}10: 00 \text { a.m. } \\
6: 30 \text { p.m. }\end{array}$ & $\dddot{i} \mathrm{day}$ & $\begin{array}{l}50 \\
50\end{array}$ & $\begin{array}{l}30 \\
30\end{array}$ & $\begin{array}{l}22.3 \\
38.7 \\
40.2\end{array}$ & $\begin{array}{l}\text { Barely breathed } \\
\text { After }\end{array}$ \\
\hline 44 & $3 / 1$ & $6: 00$ p.m. & 4 days & 50 & 30 & $\begin{array}{l}42.5 \\
40.2\end{array}$ & Before struggled \\
\hline 45 & $3 / 1$ & 6:00 p.m. & 1 day & 50 & 30 & $\begin{array}{l}44.61 \\
40.28\end{array}$ & Before struggled \\
\hline 46 & $3 / 1$ & 5:40 p.m. & 1 day & 50 & 30 & $\begin{array}{l}43.27 \\
40.28\end{array}$ & Before cried very much \\
\hline 47 & $3 / 1$ & 11:20 a.m. & 1 day & 50 & 30 & $\begin{array}{l}42.52 \\
39.54\end{array}$ & Quiet \\
\hline 48 & $3 / 1$ & 11:30 a.m. & 1 day & 50 & 30 & $\begin{array}{l}32.08 \\
32.08\end{array}$ & Right after cried \\
\hline
\end{tabular}

Table 2 shows the results of forty-eight determinations on twenty normal new-borns were made. The respiration time was 30 seconds, and the initial amount of air in the bag 50 c.c. After the first fifty readings (Table 1 ) it was decided that the amount of air in the bag used for infants (Howland) was too large for new-borns, because very often associated with low readings there was practically no change in the contour of the bag during respiration, which meant insufficient, or no diffusion, of air in the bag, with air expired. Therefore, the lowest possible amount of air in bag was used. This was one bulb 
TABLE 3.-Effect of Food on $\mathrm{CO}_{2}$ Alveolar Tension

(Twenty-Five Readings, 30 Seconds; 50 c.c. in Bag.)

\begin{tabular}{|c|c|c|c|c|c|c|c|c|c|c|}
\hline No. & Date & $\begin{array}{c}\text { Time of } \\
\text { Day }\end{array}$ & Age & Air in & $\begin{array}{l}\text { Time, } \\
\text { See. }\end{array}$ & \multicolumn{2}{|c|}{ Before } & \multicolumn{2}{|c|}{ After } & Remarks \\
\hline 1 & $12 / 11$ & 10:30 a.m. & 8 days & 150 & 30 & 30 & $\begin{array}{l}24.6 \\
26.8\end{array}$ & 30 & $\begin{array}{l}35.8 \\
\mathbf{3 5 . 8}\end{array}$ & \\
\hline 2 & $2 / 7$ & $\begin{array}{r}\text { 9:45 a.m. } \\
\text { 10:30 a.m. }\end{array}$ & $2 \mathrm{mos}$. & 150 & 30 & $\begin{array}{l}35.0 \\
36.5\end{array}$ & $6^{2} 30^{\circ}$ & $\begin{array}{l}35.8 \\
35.8\end{array}$ & 30 & \\
\hline 3 & $2 / 9$ & 12:10 p.m. & 2 mos. & 150 & 30 & 70 & 37.2 & 20 & $\begin{array}{l}35.06 \\
\mathbf{3 5 . 0 6}\end{array}$ & \\
\hline 4 & $2 / 27$ & 5:45 p.m. & 5 days & 150 & 30 & 15 & $\begin{array}{l}37.25 \\
40.28\end{array}$ & 15 & $\begin{array}{l}32.8 \\
33.5\end{array}$ & \\
\hline 5 & $2 / 28$ & $\begin{array}{l}\text { 2:50 p.m. } \\
\text { 5:45 p.m. }\end{array}$ & 12 hrs. & 150 & 30 & 10 & $\begin{array}{l}20.81 \\
22.38\end{array}$ & 50 & $\begin{array}{l}26.8 \\
24.6\end{array}$ & No food obtained \\
\hline 6 & $2 / 28$ & $\begin{array}{r}9: 45 \text { a.m. } \\
10: 15 \text { a.m. }\end{array}$ & 11 hrs. & 150 & 30 & 15 & $\begin{array}{l}25.36 \\
25.36\end{array}$ & 15 & $\begin{array}{l}20.14 \\
18.65\end{array}$ & $\begin{array}{l}\text { No gain in weight, } \\
\text { very quiet }\end{array}$ \\
\hline 7 & $2 / 28$ & $\begin{array}{l}\text { 10:00 a.m. } \\
\text { 10:30 a.m. }\end{array}$ & 3 days & 150 & 30 & 10 & $\begin{array}{l}18.65 \\
15.66\end{array}$ & 15 & $\begin{array}{l}18.65 \\
20.14\end{array}$ & $\begin{array}{l}\text { Very quiet, } 7 \text { th } \\
\text { mo. premature }\end{array}$ \\
\hline 8 & $2 / 28$ & $\begin{array}{l}\text { 10:10 a.m. } \\
10: 45 \text { a.m. }\end{array}$ & 3 days & 150 & 30 & $10^{\circ}$ & $\begin{array}{l}26.11 \\
26.11\end{array}$ & 30 & $\begin{array}{l}23.13 \\
25.36\end{array}$ & $\begin{array}{c}\text { Very quiet, 8th } \\
\text { mo, premature }\end{array}$ \\
\hline 9 & $3 / 1$ & $\begin{array}{l}9: 45 \text { a.m. } \\
10: 30 \text { a.m. }\end{array}$ & 12 hrs. & 50 & 30 & 20 & $\begin{array}{l}32.08 \\
37.3\end{array}$ & $30^{\circ}$ & $\begin{array}{l}25.3 \\
30.5\end{array}$ & $\begin{array}{l}\text { No gain in weight, } \\
\text { nursed }\end{array}$ \\
\hline 10 & $3 / 1$ & $\begin{array}{l}\text { 5:45 p.m. } \\
\text { 6:30 p.m. }\end{array}$ & 7 days & 50 & 30 & 15 & $\begin{array}{l}35.06 \\
35.06\end{array}$ & $15^{\prime}$ & $\begin{array}{l}30.59 \\
32.08\end{array}$ & Cried very much \\
\hline 11 & $3 / 2$ & $\begin{array}{l}\text { 9:50 a.m. } \\
\text { 10:10 a.m. }\end{array}$ & 2 days & 150 & 30 & $10^{\circ}$ & $\begin{array}{l}22.38 \\
21.68\end{array}$ & $10^{\prime}$ & $\begin{array}{l}29.84 \\
25.36\end{array}$ & \\
\hline 12 & $3 / 2$ & $\begin{array}{l}\text { 9:55 a.m. } \\
\text { 3:00 p.m. }\end{array}$ & 24 brs. & 150 & 30 & 5 & $\begin{array}{l}38.7 \\
35.9\end{array}$ & 30 & $\begin{array}{l}22.38 \\
25.36\end{array}$ & \\
\hline 13 & $3 / 3$ & $\begin{array}{l}\text { 9:40 a.m. } \\
\text { 5:15 p.m. }\end{array}$ & 3 days & 150 & 30 & $10^{\circ}$ & $\begin{array}{l}30.59 \\
30.59\end{array}$ & 120 & $\begin{array}{l}26.11 \\
32.82\end{array}$ & \\
\hline 14 & $3 / 3$ & $\begin{array}{l}\text { 9:40 a.m. } \\
\text { 5:15 p.m. }\end{array}$ & 3 days & 50 & 30 & & $\begin{array}{l}42.52 \\
43.27\end{array}$ & 120 & $\begin{array}{l}40.28 \\
37.30\end{array}$ & \\
\hline 15 & $3 / 4$ & $\begin{array}{l}\text { 9:50 a.m. } \\
\text { 3:40 p.m. }\end{array}$ & 5 hrs. & 50 & 30 & & $\begin{array}{l}38.7 \\
37.3\end{array}$ & 70 & $\begin{array}{l}27.6 \\
25.6\end{array}$ & \\
\hline 16 & $3 / 5$ & 9:50 a.m. & 1 day & 50 & 30 & $10^{\prime}$ & $\begin{array}{l}38.71 \\
40.28\end{array}$ & & & \\
\hline 17 & $3 / 5$ & 11:00 a.m. & 1 day & 50 & 30 & $\ldots$ & $\ldots \ldots$ & 30 & $\begin{array}{l}44.76 \\
44.76\end{array}$ & Struggled \\
\hline 18 & $3 / 5$ & $\begin{array}{r}11: 15 \text { a.m. } \\
\text { 5:30 p.m. }\end{array}$ & $1 \mathrm{day}$ & 50 & 30 & & $\begin{array}{l}26.11 \\
28.35\end{array}$ & 45 & $\begin{array}{l}37.3 \\
35.06\end{array}$ & \\
\hline 19 & $3 / 6$ & $\begin{array}{l}\text { 1:10 p.m. } \\
\text { 2:10 p.m. }\end{array}$ & 2 days & 50 & 30 & & $\begin{array}{l}37.3 \\
35.0\end{array}$ & $10^{\prime}$ & $\begin{array}{l}43.27 \\
41.78\end{array}$ & \\
\hline 20 & $3 / 6$ & $\begin{array}{l}\text { 1:10 p.m. } \\
\text { 2:10 p.m. }\end{array}$ & 2 days & 150 & 30 & & $\begin{array}{l}27.6 \\
27.6\end{array}$ & $10^{\circ}$ & $\begin{array}{l}38.09 \\
32.82\end{array}$ & \\
\hline 21 & $3 / 7$ & $\begin{array}{l}\text { 2:00 p.m. } \\
2: 20 \text { p.m. }\end{array}$ & 4 days & 50 & 30 & & $\begin{array}{l}43.27 \\
44.01\end{array}$ & $10^{\circ}$ & $\begin{array}{l}28.35 \\
26.11\end{array}$ & $\begin{array}{l}\text { Vomited, strug- } \\
\text { gled }\end{array}$ \\
\hline 21 & $3 / 7$ & 2:00 p.m. & 4 days & 150 & 30 & & $\begin{array}{l}35.07 \\
35.07\end{array}$ & $10^{\circ}$ & $\begin{array}{l}30.59 \\
29.84\end{array}$ & \\
\hline 22 & $3 / 8$ & $\begin{array}{l}\text { Noon } \\
\text { 1:50 p.m. }\end{array}$ & 5 days & 50 & 30 & & $\begin{array}{l}39.54 \\
37.3\end{array}$ & & $\begin{array}{l}42.52 \\
43.27\end{array}$ & Cried very much \\
\hline 22 & $3 / 8$ & $\begin{array}{r}9: 50 \text { a.m. } \\
10: 20 \text { a.m. }\end{array}$ & 4 days & 50 & 30 & & $\begin{array}{l}36.55 \\
39.54\end{array}$ & 20 & $\begin{array}{l}24.6 \\
28.3\end{array}$ & Very quiet \\
\hline 23 & $3 / 8$ & 1:50 p.m. & 8 days & 50 & 30 & & $\begin{array}{l}41.03 \\
41.03\end{array}$ & 10 & $\begin{array}{l}40.28 \\
40.28\end{array}$ & \\
\hline 24 & $3 / 8$ & $\begin{array}{l}\text { 5:15 p.m. } \\
\text { 6:20 p.m. }\end{array}$ & 4 days & 50 & 30 & & $\begin{array}{l}37.3 \\
41.08\end{array}$ & 20 & $\begin{array}{l}41.78 \\
42.52\end{array}$ & \\
\hline 25 & $3 / 8$ & $\begin{array}{l}2: 00 \text { p.m. } \\
\text { 4:40 p.m. }\end{array}$ & $1 \mathrm{day}$ & 50 & 30 & $\ldots$ & $\ldots \ldots$ & 120 & $\begin{array}{l}39.54 \\
40.28\end{array}$ & \\
\hline
\end{tabular}

TABle 4.-Determination of $\mathrm{CO}_{2}$ Alveolar Tension of Eight New-Borns from 1 Hour to 8 Days. Old with the Effect of Exercise on Determination

(50 c.c. in Bag; 30 Seconds.)

\begin{tabular}{|c|c|c|c|c|c|c|c|c|c|c|}
\hline No. & $\begin{array}{c}1 \text { Hour } \\
\text { to } \\
1 \text { Day }\end{array}$ & 1 Day & 2 Days & 3 Days & 4 Days & 5 Days & 6 Days & 7 Days & $\begin{array}{c}\text { From } \\
8 \text { to } 14 \\
\text { Days }\end{array}$ & Remarks \\
\hline $\begin{array}{l}1 \\
2\end{array}$ & $\begin{array}{l}41.78 \\
41.78\end{array}$ & $\begin{array}{l}40.28 \\
44.01\end{array}$ & $\begin{array}{l}29.8 \\
\ldots \ldots\end{array}$ & $\begin{array}{l}45.51 \\
42.52\end{array}$ & $\begin{array}{l}32.08 \\
40.28\end{array}$ & $\begin{array}{l}45.51 \\
\ldots \ldots\end{array}$ & $\begin{array}{l}41.03 \\
\ldots \ldots\end{array}$ & $\begin{array}{l}37.3 \\
40.28\end{array}$ & $\cdots \cdots$ & $\begin{array}{c}12 \text { hrs. } \\
32.08\end{array}$ \\
\hline $\begin{array}{l}3 \\
4\end{array}$ & $\begin{array}{l}4 \text { hrs. } \\
43.27\end{array}$ & $\begin{array}{l}38.7 \\
42.52\end{array}$ & $\begin{array}{l}41.78 \\
37.3\end{array}$ & $\begin{array}{l}38.79 \\
43.27\end{array}$ & $\begin{array}{l}37.3 \\
44.01\end{array}$ & $\begin{array}{l}41.08 \\
28.35\end{array}$ & $\begin{array}{l}40.28 \\
43.27\end{array}$ & $\begin{array}{l}40.28 \\
39.54\end{array}$ & 53.59 & $10 \mathrm{hrs}$. \\
\hline $\begin{array}{l}5 \\
6\end{array}$ & $\begin{array}{c}5 \text { hrs. } \\
38.7 \\
6 \text { hrs. } \\
40.28\end{array}$ & $\begin{array}{l}40.28 \\
\ldots \ldots\end{array}$ & $\begin{array}{l}39.54 \\
27.6\end{array}$ & 28.35 & 34.32 & & & & . & \\
\hline $\begin{array}{l}7 \\
8\end{array}$ & ..... & $\begin{array}{l}37.3 \\
\ldots \ldots\end{array}$ & ..... & $\begin{array}{l}37.3 \\
\ldots \ldots\end{array}$ & $\begin{array}{l}41.78 \\
46.25\end{array}$ & & & & & \\
\hline
\end{tabular}


full, which is the equivalent of about 50 c.c. The difference between the $\mathrm{CO}_{2}$ tension, using 50 c.c. of air in the bag, is uniformly and strikingly different from that where 150 c.c. is used. The results also are much more uniform with the former. They range from $26 \mathrm{~mm}$. to $45 \mathrm{~mm}$. The few readings lower than this can be eliminated

TABle 5.-Comparison of Readings with Different Amocnts of Air in Bag and the Determination of a Standard

\begin{tabular}{|c|c|c|c|c|c|c|}
\hline No. & 50 C.c. & 150 C.c. & 250 C.c. & Age & Time & Remarks \\
\hline 1 & $\begin{array}{l}43.27 \\
41.28\end{array}$ & $\begin{array}{l}38.09 \\
32.82\end{array}$ & $\ldots$. & 3 days & 2:10 p.m. & $\mathrm{i}$ \\
\hline 2 & $\begin{array}{l}37.3 \\
35.06\end{array}$ & 27.6 & $\ldots$. & 3 days & 1:10 p.m. & 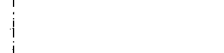 \\
\hline 3 & $\begin{array}{l}41.03 \\
48.79\end{array}$ & $\begin{array}{l}35.06 \\
29.8\end{array}$ & $\ldots$ & 5 days & 12 Noon & \\
\hline 4 & $\begin{array}{l}37.3 \\
35.06\end{array}$ & $\begin{array}{l}32.08 \\
32.08\end{array}$ & $\ldots \ldots$ & 1 day & 11:15 a.m. & Cried \\
\hline 5 & $\begin{array}{l}37.81 \\
35.81\end{array}$ & 23.87 & $\ldots$. & 3 days & 10:00 a.m. & Before \\
\hline 6 & $\begin{array}{l}44.76 \\
44.76\end{array}$ & $\begin{array}{l}35.8 \\
35.8\end{array}$ & $\cdots$ & 1 day & 11:00 a.m. & Struggled \\
\hline 7 & $\begin{array}{l}28.35 \\
26.11\end{array}$ & $\begin{array}{l}29.09 \\
28.35\end{array}$ & $\begin{array}{l}34.32 \\
31.33\end{array}$ & 11 hours & 3:20 p.m. & \\
\hline 8 & $\cdots$ & $\begin{array}{l}38.79 \\
35.81\end{array}$ & $\begin{array}{l}35.06 \\
27.60\end{array}$ & 3 days & 3:00 p.m. & Very quiet \\
\hline 9 & $\begin{array}{l}27.6 \\
25.36\end{array}$ & $\begin{array}{l}28.35 \\
27.6\end{array}$ & 22.38 & 3 hours & 3:40 p.m. & $\begin{array}{l}\text { Very shallow, } \\
\text { barely breathed }\end{array}$ \\
\hline 10 & $\begin{array}{l}38.7 \\
38.7\end{array}$ & $\begin{array}{l}30.59 \\
32.4\end{array}$ & $\begin{array}{l}30.59 \\
32.4\end{array}$ & 1 day & 6:30 p.m. & 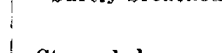 \\
\hline 11 & $\begin{array}{l}44.01 \\
42.4\end{array}$ & $\begin{array}{l}40.28 \\
35.5\end{array}$ & $\begin{array}{l}30.59 \\
30.59\end{array}$ & I day & 6:00 p.m. & Struggled \\
\hline 12 & $\begin{array}{l}43.27 \\
41.28\end{array}$ & $\begin{array}{l}40.28 \\
40.28\end{array}$ & $\begin{array}{l}30.59 \\
26.11\end{array}$ & I day & 5: $\{0$ g.m. & Struggled \\
\hline 13 & $\begin{array}{l}42.52 \\
40.28\end{array}$ & $\begin{array}{l}33.57 \\
30.59\end{array}$ & 30.59 & 1 day & 11:30 a.m. & Very quiet \\
\hline 14 & $\begin{array}{l}32.08 \\
30.59\end{array}$ & $\begin{array}{l}32.08 \\
32.08\end{array}$ & 22.38 & 1 day & 11:30 a.m. & Gried \\
\hline 15 & $\ldots$ & $\begin{array}{l}38.7 \\
35.9\end{array}$ & $\begin{array}{l}22.30 \\
20.14\end{array}$ & 24 hours & 9:55 a.m. & Cried \\
\hline 16 & $\begin{array}{l}44.01 \\
41.28\end{array}$ & $\begin{array}{l}32.08 \\
29.84\end{array}$ & $\ldots .$. & 10 days & $10: 00$ a.m. & Quiet \\
\hline 17 & $\begin{array}{l}35.06 \\
29.84\end{array}$ & $\begin{array}{l}30.59 \\
29.84\end{array}$ & $\ldots$ & 10 days & 3:00 p.m. & Quiet \\
\hline 18 & $\begin{array}{l}40.28 \\
36.55\end{array}$ & $\begin{array}{l}25.38 \\
22.38\end{array}$ & $\cdots$ & 36 hours & $3: 00 \mathrm{p.m}$ & Cried \\
\hline 19 & $\begin{array}{l}42.52 \\
43.27\end{array}$ & $\begin{array}{l}30.59 \\
30.59\end{array}$ & $\ldots$. & 3 days & 5:15 p.m. & Quiet \\
\hline 20 & $\begin{array}{l}45.51 \\
45.51\end{array}$ & $\begin{array}{l}25.36 \\
22.38\end{array}$ & $\ldots$. & 3 days & 9:50 a.m. & Quiet \\
\hline 21 & $\begin{array}{l}36.81 \\
35.81\end{array}$ & $\begin{array}{l}22.38 \\
14.92\end{array}$ & $\ldots$ & ...... & 9:56 a.m. & Barely breathed \\
\hline 22 & $\begin{array}{l}38.09 \\
37.39\end{array}$ & $\begin{array}{l}38.09 \\
36.55\end{array}$ & $\ldots$. & 6 days & 9:50 a.m. & Cried very much \\
\hline 23 & $\begin{array}{l}43.27 \\
42.52\end{array}$ & $\begin{array}{l}35.81 \\
31.33\end{array}$ & $\ldots \ldots$ & 4 hours & $9: 50$ a.m. & Struggled \\
\hline 24 & $\begin{array}{l}45.51 \\
44.61\end{array}$ & $\begin{array}{l}22.38 \\
20.89\end{array}$ & $\ldots$ & 10 hours & 4:45 p.m. & Got nothing \\
\hline 25 & $\begin{array}{l}32.08 \\
30.59\end{array}$ & $\begin{array}{l}32.82 \\
30.59\end{array}$ & $\ldots \ldots$ & 3 days & 4:50 p.m. & Oried \\
\hline 26 & $\begin{array}{l}40.28 \\
37.30\end{array}$ & $\begin{array}{l}32.82 \\
26.11\end{array}$ & $\ldots \ldots$ & 3 days & 5:15 p.m. & Quiet \\
\hline 27 & $\begin{array}{l}42.52 \\
41.28\end{array}$ & $\begin{array}{l}30.59 \\
27.62\end{array}$ & $\ldots$. & 2 days & 5:30 p.m. & Quiet \\
\hline
\end{tabular}

because in these, breathing was too shallow. The majority of the readings were from 35 to $42 \mathrm{~mm}$., and varied only from 3 to $5 \mathrm{~mm}$. Three readings were made each time, and the two within $2 \mathrm{~mm}$. of one another were recorded.

We should advise 50 c.c. of air in the bag, with 30 seconds for breathing period, as a standard for new-borns. 
Ylippo ${ }^{2}$ claims that starvation usually lowers the alveolar $\mathrm{CO}_{2}$ tension, and that ingestion of food usually increases it. We found on the other hand that food has no effect on the $\mathrm{CO}_{2}$ tension. Readings were made immediately, and one, two or three hours after feeding. The first few days, when the baby received practically no food at ail, there was no definite decrease in $\mathrm{CO}_{2}$ tension, which is indicative of acidosis, such as Ylippo claims to have found.

Ten new-borns were studied from one hour to eight days of age, 50 c.c., in the bag, 30 seconds breathing time. Although in some instances struggling and crying during the period of experiment raised the $\mathrm{CO}_{2}$ tension, yet in the majority of readings there was no change, therefore it could not be said, from our experiments, that muscular action has any definite effect on the $\mathrm{CO}_{2}$ tension.

With 250 c.c., the readings were uniformly lower than with 150 c.c., and those of 1.50 c.c., uniformly lower than 50 c.c. As to the length of time, readings taken after ten seconds of breathing showed a much lower $\mathrm{CO}_{2}$ tension than those of 20 seconds, and those of 20 seconds much lower than those of 30 seconds. Readings were taken with breathing time as high as one and one-half minutes; at which time the babies became blue.

Alkali Tolerance of Normal New-Borns.--The alkali tolerance test is a practical and quick method of determining the amount of sodium bicarbonate necessary to change the urine from acid to alkali. Since Ylippo claimed that the $P_{H}$ of the urine of normal new-borns showed high acidity we attempted to confirm his findings by this test. We administered orally with the stomach tube $0.16 \mathrm{gm}$. and $0.33 \mathrm{gm}$. of sodium bicarbonate. Sodium bicarbonate, $0.33 \mathrm{gm}$., administered every two hours, invariably caused vomiting after the first or second dose, therefore, $0.16 \mathrm{gm}$. was given every two hours which was retained by all babies. Of thirty babies, nine vomited; of the twenty-one remaining, the results as shown in the chart were obtained.

The lowest amount necessary to change the urine to alkaline was $0.1 \mathrm{gm}$., the highest, $4 \mathrm{gm}$; the average was $1.7 \mathrm{gm}$. The results would probably have been lower if one intravenous injection had been made, but this was impractical. Even though in proportion to the weight, a much smaller amount of sodium bicarbonate is necessary to change the urine of a normal new-born to alkaline than in an aduit, nevertheless, it is difficult to see how one can conclude from these results that the urine of a normal new-born shows high acidity.

2. Ylippo, A.: Ueber die $\mathrm{CO}_{2}$ dissoziations-kurve und die respiratorisclie funktion des blutes bei säuglingen mit schweren ernährungsstörungen, Berl. klin. Wchnschr. 52:252, 1915. 
A quantitated determination of the acetone in the urine of ten normal new-borns was made (Huppert-Messenger method).

Twenty-four-hour specimens were collected. In only one was acetone found; $0.3 \mathrm{mg}$. was the twenty-four hour amount. These findings do not bear out the assumption that the new-born is acidotic at birth.

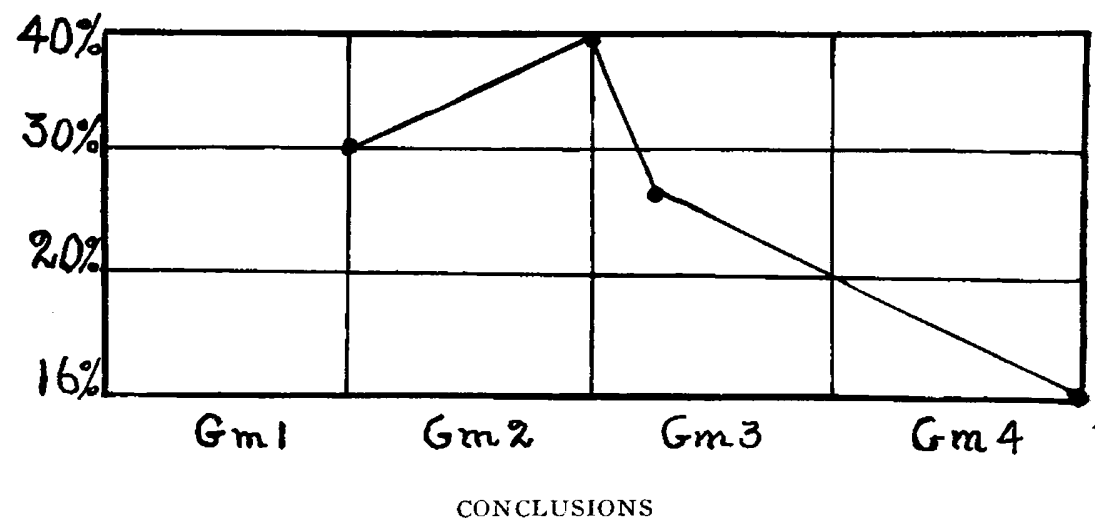

1. Alveolar $\mathrm{CO}_{2}$ tension is a practical index of acidosis.

2. Fifty c.c., of air in the bag, over a period of 30 seconds for breathing gives the most constant results.

3. The modification of Plesch-Higgins' method of collecting air with the use of the pulmotor mask is the most practical way of collecting air from new-borns.

4. We have not been able to establish a lower $\mathrm{CO}_{2}$ tension which is indicative of the so-called "acidotic state."

5. The ingestion of food, or starvation and muscular exercise under these conditions, have no constant demonstrable effect on the alveolar $\mathrm{CO}_{2}$ tension.

6. The urine of the normal new-born is nearly always acid.

7. It takes on an average $1.7 \mathrm{gm}$. of sodium bicarbonate to turn the urite from acid to alkaline, giving $0.16 \mathrm{gm}$. every two hours by mouth.

8. Our results with the alkali tolerance test for normal new-borns do not indicate an acidosis.

9. Practically no acetone is found in the urine of normal newborns.

This work has been discontinued during the period of the war, but it is hoped that it can be resumed with a view of investigating the alkali reserve and the $\mathrm{P}_{\mathrm{H}}$ of the blood of normal new-borns.

We wish to thank Dr. Rowntree of the department of medicine for his many suggestions, and Miss Mildred Ziegler, instructor in the department of pediatrics, for aid in the work. 